23

\title{
Безаберрационная ширина углового спектра зондирующего слоистый объект поля в конфокальной микроскопии
}

\author{
(С) Д.В. Лякин ${ }^{1}$, Л.А. Максимова ${ }^{1}$, В.П. Рябухо ${ }^{1,2}$ \\ ${ }^{1}$ Институт проблем точной механики и управления РАН, \\ 410028 Саратов, Россия \\ ${ }^{2}$ Саратовский национальный исследовательский государственный университет имени Н.Г. Чернышевского, \\ 410012 Саратов, Россия \\ e-mail: LDV-77@mail.ru
}

Поступила в редакцию 28.12.2018 г.

В окончательной редакции 28.12.2018 г.

Принята к публикации 15.03.2019 г.

Теоретически и экспериментально исследован эффект влияния числовой апертуры зондирующего объект пучка света на измеряемую с помощью конфокального интерференционного микроскопа величину при определении толщины слоистого объекта, для случая существенного влияния на сигнал сферической аберрации, возникающей в зондирующем объект пучке при его прохождении через объект. Получена зависимость измеряемой величины от числовой апертуры с учетом влияния на сигнал сферической аберрации. Установлен критерий „безаберрационного“ распространения зондирующего объект пучка и определено условие, при котором влиянием сферической аберрации на сигнал конфокального микроскопа можно пренебречь. Получено приближенное аналитическое выражение, позволяющее с учетом эмпирического корректирующего фактора определить „безаберрационную“ ширину углового спектра зондирующего объект пучка света. Определены границы применимости аналитической зависимости измеряемой величины от числовой апертуры, которая получена ранее для случая пренебрежимо малого влияния сферической аберрации на сигнал конфокального микроскопа.

Ключевые слова: конфокальная микроскопия, интерференционный микроскоп, сферическая аберрация, декорреляция, слоистый объект

DOI: $10.21883 /$ OS.2019.09.48211.381-18

\section{Введение}

Одной из задач, решаемых с помощью конфокальной микроскопии [1], как метода неразрушающего $3 D$ контроля геометрических и оптических параметров микрообъектов биологического и технического происхождения [2-7], является задача определения геометрической толщины тонких прозрачных и/или слабо рассеивающих объектов [8-13]. Измерение толщины слоистого прозрачного объекта с помощью конфокального микроскопа осуществляется путем сканирования объекта по глубине остро сфокусированным зондирующим пучком света. При совпадении перетяжки зондирующего пучка с границами объекта в сигнале конфокального микроскопа возникают пикообразные максимумы, ширина которых определяется числовой апертурой пучка и размером конфокальной апертуры $[1,14]$. Интервал $\delta z$ между максимумами пиков сигнала от границ однослойного объекта, в шкале его продольного смещения $\Delta z$, служит измеряемой с помощью конфокального микроскопа величиной [8-12].

Основной проблемой при определении реальной геометрической толщины исследуемого слоистого микрообъекта из результатов его измерения с помощью конфокального микроскопа является нелинейный характер зависимости измеряемой величины $\delta z$ как от параметров самого микрообъекта (показателя прелом- ления материала и геометрической толщины), так и от апертурных (угловых) параметров зондирующего объект пучка света [8-12]. Эта нелинейность проявляется тем сильнее, чем больше скачок показателя преломления на границе раздела иммерсионная среда/объект, т.е., чем сильнее отличаются показатели преломления этих двух сред. Наличие скачка показателя преломления на границе раздела иммерсионная среда/объект приводит к нарушению гомоцентричности проходящего через эту границу сходящегося пучка света, и в нем возникает сферическая аберрация [15-17] вследствие того, что лучи, распространяющиеся под различными углами к оптической оси, после преломления на указанной границе пересекают оптическую ось уже не в одной фокальной точке (номинальном фокусе), а в различных фокальных точках. В результате формируется распределение интенсивности вдоль оптической оси пучка с максимумом, положение которого не совпадает ни с фокусом преломившихся параксиальных лучей, ни с фокусом преломившихся крайних (апертурных) лучей пучка [18]. Положение этого максимума интенсивности определяет положение актуального фокуса зондирующего пучка внутри среды объекта. В случае однослойного объекта, сканируемого в конфокальном микроскопе по глубине, сигнал от задней границы слоя возникает при совпадении этой границы с актуальным фокусом преломленной волны. 
В работах по конфокальной микроскопии и по методам интерференционной корреляционной микроскопии [19-29], где также используется конфокальный режим формирования оптического сигнала, наиболее распространены следующие оценки для $\delta z$, полученные в рамках приближения параксиальных лучей [20,24-29]:

$$
\delta z_{p} \approx d n_{0} / n
$$

и в рамках приближения крайних (апертурных) лучей зондирующего объект пучка света $[10,12,30-33]$ :

$$
\delta z_{m} \approx d \sqrt{n_{0}^{2}-N A^{2}} / \sqrt{n^{2}-N A^{2}}
$$

где $d$ - геометрическая толщина слоя, $n-$ абсолютный показатель преломления вещества слоя на длине волны $\lambda_{0}$ используемого в конфокальном микроскопе лазерного источника света, $n_{0}$ - показатель преломления иммерсионной среды, соответствующий $\lambda_{0}$, $N A=n_{0} \sin \left(\theta_{\max }\right)$ - числовая апертура зондирующего объект пучка света, $\theta_{\max }$ - апертурный угол.

В [34] в предположении пренебрежимо малого влияния на сигнал конфокального микроскопа сферической аберрации, возникающей в зондирующем объект пучке света при его прохождении через границу раздела иммерсионная среда/объект, получена точная аналитическая формула, отражающая зависимость измеряемой с помощью конфокального микроскопа величины от числовой апертуры зондирующего объект пучка

$$
\delta z=d \frac{n_{0}+\sqrt{n_{0}^{2}-N A^{2}}}{\sqrt{4\left(n^{2}-n_{0}^{2}\right)+\left(n_{0}+\sqrt{n_{0}^{2}-N A^{2}}\right)^{2}}} .
$$

В настоящей работе мы представляем результаты теоретического и экспериментального исследований влияния числовой апертуры $N A$ зондирующего слоистый объект пучка монохроматического света на определяемую с помощью конфокального микроскопа величину $\delta z$, для случая существенного влияния на сигнал конфокального микроскопа сферической аберрации, возникающей в зондирующем объект пучке, и определяем границы применимости выражения (3).

\section{Влияние сферической аберрации, вносимой объектом в зондирующий пучок света, на сигнал лазерного интерференционного конфокального микроскопа}

Процесс прохождения сходящегося монохроматического пучка через границу раздела сред со скачком показателя преломления достаточно хорошо исследован теоретически и экспериментально [15-18,35]. Сферическая аберрация, возникающая в зондирующем объект пучке света при его прохождении через границу раздела иммерсионная среда - объект, влияет на ширину и амплитуду максимума осевого распределения интенсивности поля вблизи актуального фокуса преломленной волны. Чем больше эта аберрация, тем больше ширина распределения интенсивности и меньше амплитуда его максимума [15-17]. При достижении сферической аберрацией определенного порога [15] возникает сильная асимметрия распределения интенсивности вблизи актуального фокуса, появляются дополнительные боковые максимумы, величина которых становится сравнимой с величиной глобального максимума этого распределения [11,15-17]. Таким образом, наличие сферической аберрации приводит к снижению продольного разрешения конфокального микроскопа и уменьшению отношения сигнал/шум. Этот эффект тем сильнее, чем больше числовая апертура NA пучка, зондирующего объект. Однако, как это будет показано ниже, значительная сферическая аберрация приводит также к изменению положения максимума пика сигнала от задней границы объекта и несовпадению этого положения с величиной $\delta z$, определяемой выражением (3). Такое влияние сферической аберрации на сигнал конфокального микроскопа и будем определять как существенное.

Таким образом, с практической точки зрения важно установить границы применимости выражения (3) для определения параметров слоистого объекта с помощью конфокального микроскопа. Для этого необходимо определить ширину $\Delta \widetilde{\theta}$ углового спектра (и соответствующую этой ширине числовую апертуру $N A_{\text {nonab }}$ ) зондирующего слоистый объект пучка, при которой влияние на сигнал микроскопа сферической аберрации, возникающей в зондирующем объект пучке, можно считать несущественным. В дальнейшем такую ширину углового спектра будем называть „безаберрационной“, а режим распространения зондирующего объект пучка „безаберрационным“.

Для теоретического и экспериментального исследования границ применимости формулы (3) и определения „безаберрационной“ ширины углового спектра зондирующего слоистый объект поля, как и в работе [34], использовалась схема оптического когерентного микроскопа $[28,34,36]$ (Int1 на рис. 1) с лазерным источником LS, генерирующим квазимонохроматическое излучение на длине волны $\lambda_{0}$. Микроскоп Int1 является разновидностью корреляционного микроскопа [21], в котором максимум интерференционного сигнала достигается при согласовании волновых фронтов объектной и опорной волн на выходе интерферометра, возникающем при совпадении перетяжки зондирующего объект пучка с границами раздела слоя [36]. Микроскоп Int1 может быть рассмотрен в качестве интерференционного конфокального микроскопа, в котором перетяжка опорного пучка на выходе интерферометра играет роль виртуальной конфокальной апертуры [1,21]. Схема лазерного интерференционного корреляционного микроскопа Int1 была выбрана в качестве основы данного исследования, поскольку в последнее время наблюдается интерес к 


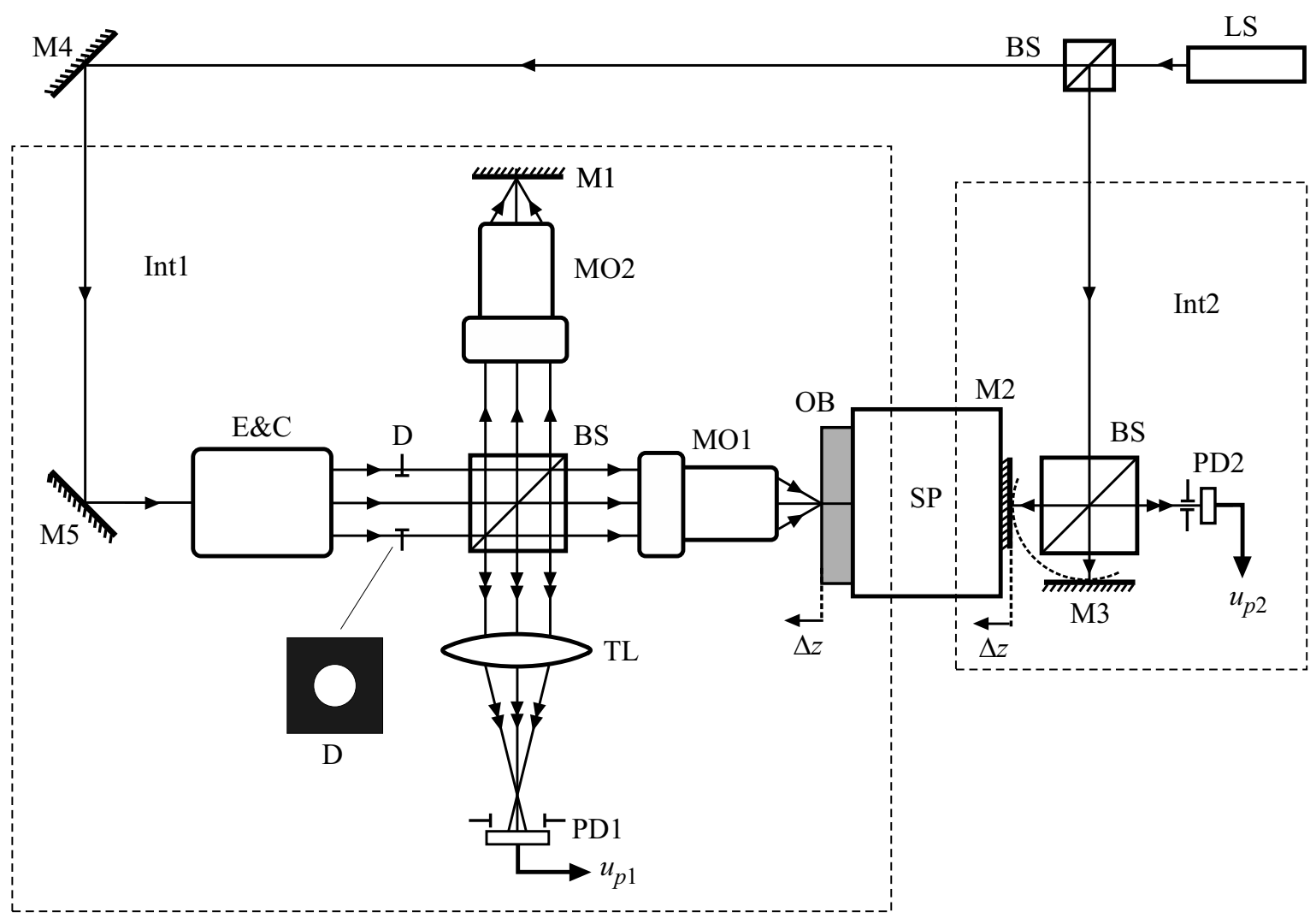

Pис. 1. Оптическая схема экспериментальной установки: Int 1 - интерференционный микроскоп; Int 2 - вспомогательный интерферометр; LS - лазерный источник света; BS - светоделитель; MO1, MO2 - идентичные микрообъективы; ОВ слоистый объект; M1-M5 - зеркала; SP - электромеханический сканер; Е \& C - расширитель и коллиматор пучка; D апертурная диафрагма; TL - тубусная линза; PD1, PD2 - фотодетекторы; $u_{p 1}, u_{p 2}$ - сигналы фотодетекторов; $\Delta z-$ продольное смещение объекта ОВ и зеркала М2.

корреляционной микроскопии с использованием частотно узкополосных (лазерных) источников света [29,3740], как альтернативы корреляционным интерференционным микроскопам с частотно широкополосными источниками света [19-28]. Использование частотно узкополосного источника снимает вопрос влияния дисперсии материала исследуемого объекта на сигнал корреляционного интерференционного микроскопа и необходимости компенсации так называемого эффекта дефокусировки, возникающего при использовании частотно широкополосного излучения в сочетании с высокоапертурными объективами [24-28], снижает требования к хроматической коррекции используемых оптических элементов и юстировке (выравниванию) длин объектного и опорного плеч интерферометра.

Сканирование объекта по глубине в интерференционном микроскопе Int1 (рис. 1) осуществляется с помощью электромеханического сканера SP. Вспомогательный интерферометр (Int2), одно из зеркал которого (M2) механически жестко связано со сканирующей платформой SP и в который направляется часть узкого пучка излучения лазера LS, используется для определения величины $\delta z$ в сигнале измерительного микроскопа Int1. Одновременная регистрация сигналов $u_{p 1}(\Delta z)$ и $u_{p 2}(\Delta z)$ интерферометров Int1 и Int2 позволяет при известной длине волны $\lambda_{0}$ источника LS перевести шкалу дискретных отсчетов сигнала интерферометра Int1 в шкалу продольного смещения $\Delta z$ объекта ОВ в микрометрах [34].

В эксперименте максимальная ширина углового спектра зондирующего слоистый объект поля определялась числовой апертурой $N A$ идентичных объективов MO1 и MO2 в плечах интерферометра Int1 при условии полного заполнения их круговых апертур (зрачков) коллимированным и расширенным пучком излучения лазера LS. Апертурная диафрагма D на рис. 1 условно соответствует собственным круговым апертурам объективов MO1 и МO2.

На рис. 2 представлены огибающие интерференционных сигналов от однослойных объектов - покровных стекол Menzel-Gläser различной геометрической толщины $d$, марка стекла Schott D263, показатель преломления для длины волны $\lambda_{0}=633 \mathrm{~nm} \mathrm{He-Ne-}$ лазера, использовавшегося в качестве источника LS, $n\left(\lambda_{0}\right)=1.5213$ [41]. Числовая апертура $N A$ зондирующего пучка света при максимальном заполнении апертуры объектива MO1 (Микромед LMPlan 50x/0.55, коррекция на бесконечность без покровного стекла) была определена независимым способом и составила $0.502 \pm 0.001$ для $\lambda_{0}=633 \mathrm{~nm}$. 
На рис. 2 отчетливо видна деградация пика сигнала от задней границы раздела слоистого объекта с ростом его толщины - уменьшение величины глобального максимума этого пика, его уширение и появление дополнительных локальных максимумов. Данная деградация является проявлением декорреляционных процессов в зондирующем объект пучке как следствия возрастающей с увеличением толщины $d$ объекта сферической аберрации. Вертикальными пунктирными линиями на рис. 2, $a-$ $d$ отмечены значения величины $\delta z$, определяемой формулой (3) для указанной выше числовой апертуры объектного пучка и соответствующей параметрам объекта (геометрической толщине $d$ и показателю преломления $n)$. Видно, что на рис. $2, b-d$ положение глобального максимума пика сигнала от задней границы объекта перестает совпадать со значениями $\delta z$, рассчитанными по формуле (3), что ограничивает возможность применения этой формулы в случае существенного влияния сферической аберрации на сигнал интерференционного конфокального микроскопа.

\section{Критерий безаберрационного распространения зондирующего объект пучка}

Переменная составляющая фотоэлектрического сигнала интерференционного микроскопа Int1 в случае однослойного объекта может быть представлена в виде $[28,34,42]$

$$
u_{p 1}(\Delta z) \sim \operatorname{Re}\left\{\Gamma_{1}(\Delta z)+\Gamma_{2}(\Delta z)\right\}
$$

где $\Gamma_{1}(\Delta z)$ и $\Gamma_{2}(\Delta z)$ - комплекснозначные функции взаимной продольной корреляции опорного поля и частей объектного поля, отразившихся от передней и задней границ объекта.

Функции корреляции $\Gamma_{1}(\Delta z)$ и $\Gamma_{2}(\Delta z)$ определяются выражениями вида $[23,24,28,42,43]$

$$
\Gamma_{j}(\Delta z) \sim \int_{k_{z \min }}^{k_{z} \max } w_{j}\left(k_{z}\right) \exp \left\{i k_{z} 2 \Delta z\right\} d k_{z}, \quad j=1,2,
$$

где

$$
k_{z}(\theta)=\frac{2 \pi}{\lambda_{0}} n_{0} \cos (\theta)
$$

- продольная пространственная частота поля $[23,24,28,42,43]$, соответствующая лучевой компоненте, распространяющейся в иммерсионной среде с показателем преломления $n_{0}$ под углом $\theta$ к оптической оси $Z$; $k_{z \min }$ и $k_{z \max }-$ минимальная и максимальная продольные пространственные частоты, соответствующие углам распространения $\theta=\theta_{\max }$ и $\theta=\theta_{\min } ; w_{j}\left(k_{z}\right)-$ взаимные спектры продольных пространственных частот опорного поля и частей зондирующего объект поля, отразившихся от передней и задней границ объекта

$$
w_{1}\left(k_{z}\right) \sim r_{1}\left(k_{z}\right) w_{0}\left(k_{z}\right)
$$
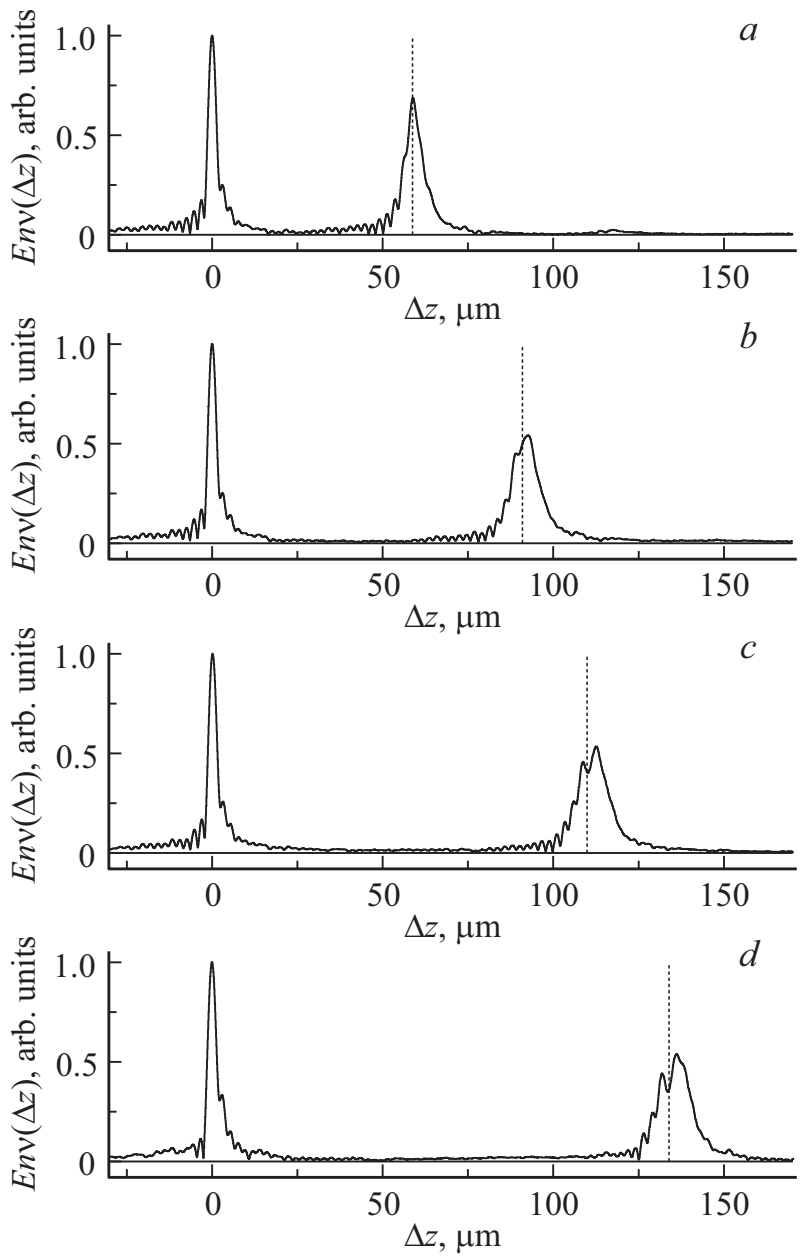

Рис. 2. Огибающие (коэффициенты модуляции) интерференционных сигналов от однослойных микрообъектов - покровных стекол различной толщины $d: 93 \pm 1(a), 144 \pm 1(b)$, $174 \pm 1(c), 212 \pm 1 \mu \mathrm{m}(d)$.

$$
w_{2}\left(k_{z}\right) \sim r_{2}\left(k_{z}\right) w_{0}\left(k_{z}\right) \exp \left\{-i \Phi\left(k_{z}\right)\right\},
$$

где $r_{j}\left(k_{z}(\theta)\right)=r_{j}(\theta)$ - коэффициенты, учитывающие отражение полей от передней и задней границ объекта и опорного зеркала M1 $\left(r_{1}(\theta)+r_{2}(\theta)=1\right)$; $w_{0}\left(k_{z}(\theta)\right) \sim I(\theta) k_{z}(\theta)$ - спектр продольных пространственных частот опорного поля; $I(\theta)$ - угловое распределение интенсивности поля во входных зрачках одинаковых микрообъективов MO1 и MO2; $\Phi\left(k_{z}\right)$ - фазовый набег, обусловленный прохождением части объектного поля через однослойный объект и определяемый с точностью до возможных фазовых скачков при отражении от границ раздела слоистого объекта выражением [42]

$$
\Phi\left(k_{z}\right)=2 d \sqrt{\left(\frac{2 \pi}{\lambda_{0}}\right)^{2}\left(n^{2}-n_{0}^{2}\right)+k_{z}^{2}} .
$$

В предположении, что коэффициенты $r_{1}\left(k_{z}\right)$ и $r_{2}\left(k_{z}\right)$ не зависят от углов распространения $\theta$ лучевых компонент опорного и объектного полей, влияние объекта (слоя) на прошедшее через него поле и соответственно 
на взаимный спектр $w_{2}\left(k_{z}\right)$ сводится к фазовым искажениям, вносимым фазовым набегом $\Phi\left(k_{z}\right)$. Фазовые искажения взаимного спектра $w_{2}\left(k_{z}\right)$ приводят к амплитудно-фазовым искажениям функции корреляции $\Gamma_{2}(\Delta z)$, что отражается на пике сигнала от задней границы объекта.

В работе [34] показано, что положение максимума пика интерференционного сигнала от задней границы раздела однослойного объекта и соответственно интервал $\delta z$ между максимумами пиков сигнала от обеих границ объекта определяется в шкале продольного смещения $\Delta z$ объекта некоторой средней продольной пространственной частотой $k_{z 0}$

$$
\delta z=d k_{z 0} / \sqrt{\left(\frac{2 \pi}{\lambda_{0}}\right)^{2}\left(n^{2}-n_{0}^{2}\right)+k_{z 0}^{2}},
$$

которая в свою очередь соответствует некоторому „безаберрационному“ диапазону продольных пространственных частот зондирующего объект пучка $\Delta \widetilde{k}_{z}=\left|k_{z 1}-k_{z 2}\right|$, т. е. такому диапазону $\Delta k_{z}$ объектного поля, угловые компоненты которого из диапазона углов $\left(\theta_{1}, \theta_{2}\right)$, границам которого соответствуют продольные частоты $k_{z 1}$ и $k_{z 2}$, распространяются в объекте без существенной сферической аберрации. Для равномерного спектра продольных пространственных частот $w_{0}\left(k_{z}(\theta)\right)=$ const, средняя продольная пространственная частота определяется выражением

$$
k_{z 0}=\left(k_{z 1}+k_{z 2}\right) / 2 \text {. }
$$

В [34] для круговой апертуры зондирующего пучка с осесимметричным распределением интенсивности $I(\theta)$ предполагалось, что значение числовой апертуры $N A$ пучка и параметры объекта $d$ и $n$ таковы, что данный „безаберрационный“ диапазон $\Delta \widetilde{k}_{z}$ совпадает с шириной $\Delta k_{z \max }$ спектра продольных пространственных частот, задаваемой числовой апертурой $N A$, т. е. $\theta_{1}=\theta_{\min }=0$ и $\theta_{2}=\theta_{\max }=\arcsin \left(N A / n_{0}\right)$. С учетом предположения о равномерности спектра продольных пространственных частот $w_{0}\left(k_{z}(\theta)\right)=$ const была получена аналитическая формула (3).

Эксперимент отчетливо показывает (рис. 2), что при фиксированном значении числовой апертуры NA зондирующего пучка в зависимости от параметров объекта (например, его толщины $d$ ) могут возникать условия, при которых данная аналитическая формула будет определять положение максимума пика сигнала от задней границы слоистого объекта, а также условия, при которых эта формула не будет работать.

Для установления условий применимости формулы (3) и определения „безаберрационной“ ширины спектра пространственных частот в зондирующем объект пучке рассмотрим кольцевую апертуру зондирующего объект гомоцентрического пучка, которая характеризуется средней продольной пространственной частотой $k_{z 0}$, определяемой углом $\widetilde{\theta}_{\text {eff, и шириной спектра про- }}$ дольных пространственных частот $\Delta \widetilde{k}_{z}$, где максимальная и минимальная частоты этого диапазона частот

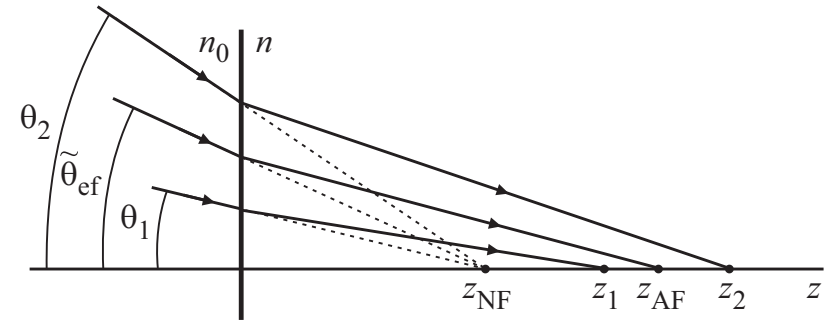

Рис. 3. Прохождение гомоцентрического сходящегося пучка с кольцевой апертурой через границу раздела сред с разными показателями преломления.

определяются углами $\theta_{1}$ и $\theta_{2}$. Для простоты и возможности соотнесения результатов данного исследования с ранее полученными результатами будем так же, как и в [34], полагать, что спектр продольных пространственных частот $w_{0}\left(k_{z}\right)$ зондирующего объект пучка с кольцевой апертурой - равномерный.

На рис. 3 приведена схема прохождения лучей, распространяющихся под углами $\widetilde{\theta}_{\mathrm{eff}}, \theta_{1}$ и $\theta_{2}$ к оптической оси, через границу раздела иммерсионная среда объект, на которой имеется скачок показателя преломления в силу различия показателей преломления иммерсионной среды $n_{0}$ и среды объекта $n$ (на рис. 3 ход лучей соответствует случаю $\left.n_{0}<n\right)$. В отсутствие этой границы раздела все три луча пересекали бы оптическую ось $Z$ в одной точке - номинальном фокусе, положение которого относительно границы раздела, принимаемой за начало отсчета вдоль оси $Z$, определяется координатой $z_{N F}$. При наличии границы раздела сред лучи, распространяющиеся под углами $\tilde{\theta}_{\mathrm{eff}}, \theta_{1}$ и $\theta_{2}$ в иммерсионной среде, после преломления на этой границе пересекут ось $Z$ в точках с координатами $z_{A F}, z_{1}$ и $z_{2}$ соответственно. Значения этих координат определяются из геометрических построений формулой вида

$$
z_{i}=z_{N F} \sqrt{n^{2}-n_{0}^{2} \sin ^{2}\left(\theta_{i}\right)} / \sqrt{n_{0}^{2}-n_{0}^{2} \sin ^{2}\left(\theta_{i}\right)} .
$$

При сканировании интерференционным микроскопом Int1 слоистого объекта по глубине, за счет продольного (осевого, вдоль оптической оси $Z$ ) смещения $\Delta z$ объекта относительно перетяжки зондирующего пучка, положение $z_{N F}$ номинального фокуса пучка определяется этим смещением, $z_{N F}=\Delta z$. При $\Delta z=0$ перетяжка зондирующего объект пучка совпадает с передней границей объекта.

Координата $z_{A F}$ определяет актуальное положение фокуса (которое считаем совпадающим с максимумом распределения интенсивности вдоль оси $Z$ ) преломившегося пучка в среде объекта, а величина $\left|z_{1}-z_{2}\right|$ будет характеризовать продольную сферическую аберрацию, возникающую в зондирующем объект пучке после его преломления на передней границе объекта. Эта аберрация приводит к декорреляции зондирующего объект поля [44], что проявляется в деградации интерференционного сигнала при увеличении глубины зондирования 
объекта (при увеличении его толщины, как в случае, показанном на рис. 2), поскольку, согласно выражению (12), величина $\left|z_{1}-z_{2}\right|$ прямо пропорциональна $z_{N F}=\Delta z$.

Максимум пика сигнала интерференционного микроскопа Int1 от задней границы раздела слоистого объекта, положение которой в выбранной системе координат вдоль оптической оси $Z$ определяется координатой $z=d$, возникает при $z_{N F}=\Delta z=\delta z$, что соответствует совпадению актуального фокуса с задней границей объекта, $z_{A F}=d$. Осевые сдвиги $\Delta z_{i}$ объекта, соответствующие совпадению фокальных точек крайних лучей пучка с задней границей раздела объекта $\left(z_{i}=d, i=1,2\right)$, определяются, согласно (12), выражением вида

$$
\Delta z_{i}=d \sqrt{n_{0}^{2}-n_{0}^{2} \sin ^{2}\left(\theta_{i}\right)} / \sqrt{n^{2}-n_{0}^{2} \sin ^{2}\left(\theta_{i}\right)} .
$$

Эффект декорреляции (декогерентности) оптического поля с широким угловым спектром при его прохождении через границы раздела прозрачных сред исследовался в [44]. В этой работе было выдвинуто предположение, что существенная декорреляция в объектном поле возникает, когда величина $\left|\Delta z_{1}-\Delta z_{2}\right|$, определяемая продольными сдвигами $\Delta z_{1}$ и $\Delta z_{2}$, соответствующими крайним лучам в пучке, становится равной длине продольной пространственной корреляции $\rho_{\|}$зондирующего объект поля, которая для равномерного спектра продольных пространственных частот определяется выражением [43]

$$
\rho_{\|}=\frac{2 \pi}{\Delta k_{z}}
$$

Взяв за основу данное предположение, будем искать аналитическое выражение для „безаберрационной“ ширины $\Delta \widetilde{k}_{z}$ спектра продольных частот и соответствующей ей „безаберрационной“ ширины углового спектра $\Delta \widetilde{\theta}=\theta_{2}-\theta_{1}$ из уравнения вида

$$
\left|\Delta z_{1}-\Delta z_{2}\right|=\rho_{\|}
$$

Для упрощения выкладок избавимся в (13) от квадратных корней, заменив их на суммы первых двух слагаемых разложения в ряд Маклорена

$$
\Delta z_{i} \approx d \frac{n_{0}}{n}\left(1-\frac{1}{2} \sin ^{2}\left(\theta_{i}\right)\right) /\left(1-\frac{1}{2} \frac{n_{0}^{2}}{n^{2}} \sin ^{2}\left(\theta_{i}\right)\right) .
$$

Возвращаясь к рассмотрению случая круговой апертуры зондирующего объект пучка, положив $\theta_{1}=0$, для величины $\left|\Delta z_{1}-\Delta z_{2}\right|$, используя (16), можно получить следующее выражение:

$$
\left|\Delta z_{1}-\Delta z_{2}\right| \approx d \frac{n_{0}}{n}\left|n^{2}-n_{0}^{2}\right| \frac{1-\cos ^{2}\left(\theta_{2}\right)}{2 n^{2}-n_{0}^{2}+n_{0}^{2} \cos ^{2}\left(\theta_{2}\right)} .
$$

Длина продольной корреляции $\rho_{\|}$в случае круговой апертуры пучка определяется выражением [43]

$$
\rho_{\|} \approx \frac{\lambda_{0}}{n_{0}\left(1-\cos \left(\theta_{2}\right)\right)} .
$$

При подстановке (17) и (18) в (15) получаем уравнение третьей степени относительно $\cos \theta_{2}$, которое имеет только одно физически реализуемое решение, исходя из условия

$$
0<\cos \left(\theta_{2}\right)<1
$$

Это решение можно получить, используя формулы Кардано для кубического уравнения [45], и оно будет иметь вид

$$
\cos \left(\theta_{2}\right)=-\frac{u+v}{2}-\frac{u-v}{2} i \sqrt{3}+\frac{1}{3}\left(\frac{\lambda_{0}}{d\left|n^{2}-n_{0}^{2}\right|}+1\right),
$$

где

$$
\begin{aligned}
& u=\left(-(q / 2)+\sqrt{(p / 3)^{3}+(q / 2)^{2}}\right)^{1 / 3}, \\
& v=\left(-(q / 2)-\sqrt{(p / 3)^{3}+(q / 2)^{2}}\right)^{1 / 3},
\end{aligned}
$$

и в свою очередь

$$
\begin{gathered}
p=-1-\frac{1}{3}\left(\frac{\lambda_{0}}{d\left|n^{2}-n_{0}^{2}\right|}+1\right)^{2}, \\
q=\frac{2}{3}-\frac{2}{27}\left(\frac{\lambda_{0}}{d\left|n^{2}-n_{0}^{2}\right|}+1\right)^{3} \\
-\frac{\lambda_{0}}{d\left|n^{2}-n_{0}^{2}\right|}\left(\frac{1}{3}+\frac{n\left(2 n^{2}-n_{0}^{2}\right)}{n_{0}^{2}}\right) .
\end{gathered}
$$

Аналитическое выражение (20), хоть и не является точным решением уравнения (15) в силу использовавшегося приближения (16), дает, как это будет показано ниже, возможность провести достаточно точную оценку „безаберрационной“ ширины $\Delta \widetilde{\theta}$ углового спектра зондирующего объект пучка с круговой апертурой при заданных параметрах объекта $d$ и $n$ и иммерсионной среды $n_{0}$.

На рис. 4, а приведено сравнение величины „безаберрационной“ числовой апертуры зондирующего объект пучка $N A_{\text {nonab }}=n_{0} \cdot \sin \left(\theta_{2}\right)$, как функции толщины $d$ исследуемого объекта, полученных путем численного решения уравнения (15) без использования приближения (16) (сплошная линия), со значениями этой величины, полученными с использованием приближенного аналитического выражения (20) (штриховая линия).

Из рис. 4, $a$ видно, что приближенная формула (20) дает завышенные значения для „безаберрационной“ числовой апертуры $N A_{\text {nonab }}$ по сравнению с точным решением, причем эта ошибка в значениях, как и ожидается, тем больше, чем меньше скачок преломления на границе иммерсионная среда/объект. Так, например, при $n_{0}=1$ и $n=1.5$ относительная ошибка $R E$ определения „безаберрационной“ числовой апертуры $N A_{\text {nопа }}$ при использовании выражения (20) не превышает $15 \%$, а при $n_{0}=1.33$ и $n=1.5$ стремиться к $44 \%$ (рис. $4, b)$.

В результате исследований получен эмпирический корректирующий фактор, позволяющий в значительной 

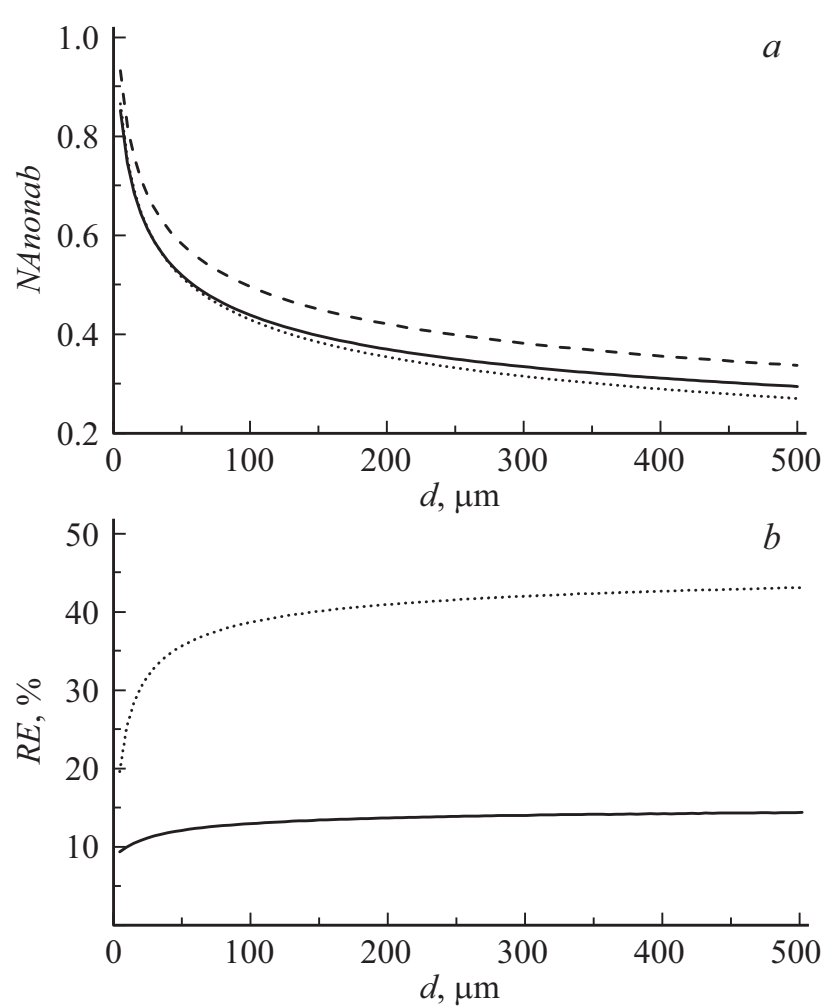

Pис. 4. К оценке точности приближенного аналитического выражения (20): $a-$ значения величины „безаберрационной“ числовой апертуры зондирующего объект пучка, полученные путем численного решения уравнения (15) (сплошная линия); значения, полученные с использованием выражения (20) (штриховая линия); скорректированные значения (пунктирная линия) при $n_{0}=1$ и $n=1.5 ; b-$ относительная ошибка $R E$ определения „безаберрационной“ числовой апертуры при использовании выражения (20) при $n_{0}=1$ и $n=1.5$ (сплошная линия) и при $n_{0}=1.33$ и $n=1.5$ (пунктир).

степени уменьшить эту ошибку. С учетом этого фактора скорректированное значение для ,.безаберрационной“ числовой апертуры зондирующего объект пучка приобретает вид

$$
N A_{\text {nonabC }} \approx N A_{\text {nonab }}-0.1 n_{0}^{2} / n .
$$

На рис. 4, $a$ в виде пунктирной линии приведен график, отображающий скорректированные значения для „.безаберрационной“ числовой апертуры.

Таким образом, с учетом эмпирического корректирующего фактора для „безаберрационной“ ширины углового спектра зондирующего объект пучка с круговой апертурой можно записать выражение

$$
\begin{aligned}
\Delta \widetilde{\theta}_{C} & =\theta_{2 C} \approx \arcsin \left(N A_{\text {nonabC }} / n_{0}\right) \\
& =\arcsin \left(\sin \left(\theta_{2}\right)-0.1 n_{0} / n\right),
\end{aligned}
$$

где $\theta_{2}$ определяется из выражения (20).

Соответственно „безаберрационная“ ширина спектра продольных пространственных частот и соответствую- щая этому диапазону частот средняя продольная пространственная частота будут определяться выражениями вида

$$
\begin{gathered}
\Delta \widetilde{k}_{z}=\left|k_{z 1}-k_{z 2}\right|=\frac{2 \pi}{\lambda_{0}} n_{0}\left(1-\cos \left(\theta_{2 C}\right)\right), \\
\widetilde{k}_{z 0}=\left(k_{z 1}+k_{z 2}\right) / 2=\frac{\pi}{\lambda_{0}} n_{0}\left(1+\cos \left(\theta_{2 C}\right)\right) .
\end{gathered}
$$

При подстановке (28) в (10) можно получить выражение, определяющее интервал между максимумами пиков сигнала интерференционного микроскопа, соответствующих передней и задней границам объекта, по своему виду аналогичное выражению (3)

$$
\delta \widetilde{z}=d \cdot \frac{n_{0}+\sqrt{n_{0}^{2}-N A_{n o n a b C}^{2}}}{\sqrt{4\left(n^{2}-n_{0}^{2}\right)+\left(n_{0}+\sqrt{n_{0}^{2}-N A_{\text {nonabC }}^{2}}\right)^{2}}}
$$

где в отличие от выражения (3) величина $N A_{\text {nonabC }}$ является функцией величин $d, n$ и $n_{0}$, т.е. зависит не только от параметров оптической системы, формирующей зондирующий объект пучок света, но и от геометрических и оптических параметров объекта.

\section{Обсуждение полученных результатов}

Выражение (29) может быть применено для более точного, чем с помощью выражения (3), определения положения максимума пика сигнала от задней границы исследуемого объекта в случае, когда исследуемый объект вносит в зондирующий пучок существенную сферическую аберрацию. На рис. 5, $a-c$ приведены огибающие интерференционных сигналов, которые показаны на рис. $2, b-d$, где в отличие от рис. 2 вертикальными пунктирными линиями отмечены значения $\delta \widetilde{z}$, рассчитанные по формуле (29). Видно, что эти значения лучше совпадают с положениями максимумов сигналов от задних границ объектов, чем значения, рассчитанные по формуле (3) при фиксированном значении числовой апертуры зондирующего объект пучка $(N A=0.502 \pm 0.001)$.

Выражение (25) определяет верхнюю границу числовой апертуры NA зондирующего объект поля, при которой сферическая аберрация, возникающая в этом поле при его прохождении через объект при заданных параметрах $d, n$ и $n_{0}$, не оказывает существенного влияния на сигнал конфокального микроскопа от задней границы объекта. Соответственно для возможности применения аналитической формулы (3) при измерении толщины прозрачных объектов методами конфокальной микроскопии следует установить следующий критерий для числовой апертуры зондирующего объект поля:

$$
N A \leq N A_{\text {nonabC }} .
$$

Таким образом, с учетом результатов данного исследования зависимость интервала между максимумами 

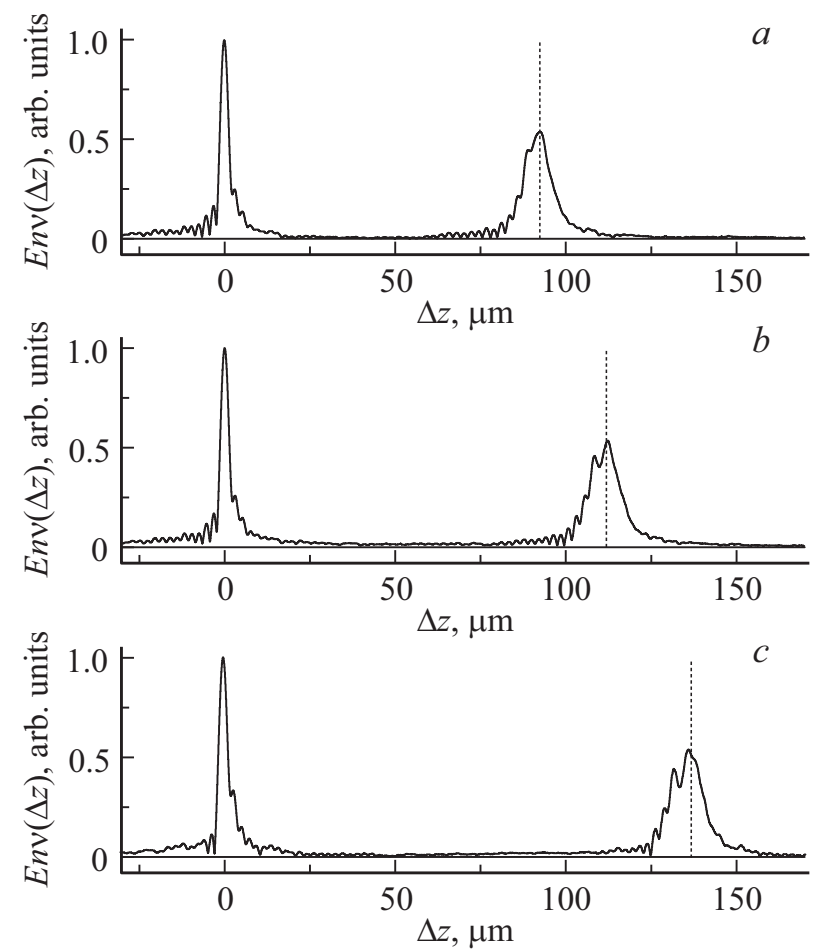

Рис. 5. Огибающие (коэффициенты модуляции) интерференционных сигналов от однослойных микрообъектов, аналогичные приведенным на рис. $2, b-d$, с положениями максимумов пиков от задних границ объектов, определенными по формуле (29) (вертикальные пунктирные линии).

пиков сигнала конфокального микроскопа, соответствующих передней и задней границам объекта, от числовой апертуры зондирующего объект пучка должна быть записана в следующем виде:

$$
\delta z(N A)=\left\{\begin{array}{c}
d \cdot \frac{n_{0}+\sqrt{n_{0}^{2}-N A^{2}}}{\sqrt{4\left(n^{2}-n_{0}^{2}\right)+\left(n_{0}+\sqrt{n_{0}^{2}-N A^{2}}\right)^{2}}} \\
\text { при } N A<N A_{\text {nonabC }}, \\
d \cdot \frac{n_{0}+\sqrt{n_{0}^{2}-N A_{\text {nonabC }}^{2}}}{\sqrt{4\left(n^{2}-n_{0}^{2}\right)+\left(n_{0}+\sqrt{\left.n_{0}^{2}-N A_{\text {nonab }}^{2}\right)^{2}}\right.}} \\
\text { при } N A \geq N A_{\text {nonabC }} .
\end{array}\right.
$$

Выражение (31) показывает, что при увеличении числовой апертуры $N A$ зондирующего объект пучка наступает момент, когда величина $\delta z(N A)$ перестает зависеть от значения этой апертуры и выходит на некий постоянный уровень, определяемый выражением (29). Причем, как показывают численные расчеты, значение величины $\delta \widetilde{z}$ с ростом $d$ асимптотически стремится к значению величины $\delta z_{p}$, определяемой выражением (1).

Влияние сферической аберрации, возникающей в зондирующем объект пучке после его прохождения границы иммерсионная среда/объект, проявляется не только в изменении положения максимума сигнала от задней границы объекта относительно значения, определяемого формулой (3), но и в уширении пика этого сигнала, уменьшению его амплитуды и появлению побочных максимумов достаточно большой амплитуды (рис. 2). „Безаберрационная“ ширина $\Delta \widetilde{k}_{z}$ спектра продольных пространственных частот зондирующего объект поля, задаваемая выражением (27), определяет, согласно выражению (14), минимальную длину продольной корреляции поля и соответственно максимально возможное для объекта и иммерсионной среды с заданными параметрами $d, n$ и $n_{0}$ продольное (по глубине) разрешение конфокального микроскопа.

\section{Заключение}

В работе исследован эффект влияния числовой апертуры зондирующего объект пучка света на результат измерения толщины слоистого объекта с помощью конфокального интерференционного микроскопа с частотно узкополосным источником света для случая существенного влияния на сигнал микроскопа сферической аберрации, возникающей в зондирующем объект пучке света при его прохождении через границу раздела иммерсионная среда/объект. Показано, что значительная сферическая аберрация приводит не только к уменьшению амплитуды интерференционных осцилляций в пике сигнала от задней границы объекта, уширению этого пика и появлению побочных максимумов достаточно большой амплитуды, но и к изменению положения глобального максимума этого пика относительно величины $\delta z$, определяемой выражением (3), полученным ранее [34].

В работе получена зависимость измеряемой с помощью конфокального микроскопа величины $\delta z$ при определении толщины слоистого объекта от числовой апертуры с учетом влияния на сигнал сферической аберрации. Данная зависимость показывает, что в случае возникновения в зондирующем объект пучке существенной сферической аберрации измеряемая величина $\delta z$ перестает зависеть от значения числовой апертуры этого пучка и выходит на некий постоянный уровень, который с ростом геометрической толщины объекта стремиться к значению, определяемому параксиальным приближением.

Установлен критерий „безаберрационного“ распространения зондирующего объект пучка и определено условие, при котором влиянием сферической аберрации на сигнал конфокального микроскопа можно пренебречь. Получено приближенное аналитическое выражение (20), позволяющее с учетом эмпирического корректирующего фактора определить „безаберрационную“ ширину углового спектра зондирующего объект пучка света (выражение $(26))$ и определить верхнюю границу применимости аналитической формулы (3).

\section{Финансирование работы}

Работа выполнена при поддержке Российского научного фонда (проект № 16-19-10528). 


\section{Конфликт интересов}

Авторы заявляют, что у них нет конфликта интересов.

\section{Список литературы}

[1] Confocal Microscopy / Ed. by Wilson T. San Diego: Academic Press, 1990. 426 p.

[2] Handbook of Biological Confocal Microscopy / Ed. by Pawley J.E. 3rd ed. Berlin: Springer, 2006. 985 p.

[3] Prasad V., Semwogerere D., Weeks E.R. // J. Phys. Cond. Matter. 2007. V. 19. P. 113102. doi 10.1088/09538984/19/11/113102

[4] Hovis D.B., Heuer A.H. // J. Microsc. 2010. V. 240. N 3. P. 173. doi 10.1111/j.1365-2818.2010.03399.x

[5] Shah S.M., Crawshaw J.P., Boek E.S. // J. Microsc. 2017. V. 265. N 2. P. 261. doi $10.1111 /$ jmi.12496

[6] Optical Inspection of Microsystems / Ed. by Osten W. NY:: SRC Press Taylor \& Francis Group, 2007. 503 p.

[7] Reyes D.R., Halter M., Hwang J. // J. Microsc. 2015. V. 259. N 1. P. 26. doi 10.1111/jmi.12245

[8] Corle T.R., Fanton J.T., Kino G.S. // Appl. Opt. 1987. V. 26. N 12. P. 2416. doi 10.1364/AO.26.002416

[9] Sheppard C.J.R., Connolly T.J., Lee J., Cogswell C.J. // Appl. Opt. 1994. V. 33. N 4. P. 631. doi 10.1364/AO.33.000631

[10] Saloma C., Matsuoka K., Kawata S. // Rev. Sci. Instrum. 1996. N 67. P. 2072. doi 10.1063/1.1147017

[11] Cox G., Sheppard C.J.R. // Micron. 2001. V. 32. P. 701. doi 10.1016/S0968-4328(01)00017-8

[12] Ilev I.K., Waynant R.W., Byrnes K.R., Anders J.J. // Opt. Lett. 2002. V. 27. N 19. P. 1693. doi 10.1364/OL.27.001693

[13] Kuypers L.C., Decraemer W.F., Dirckx J.J.J., Timmermans J.P. // J. Microsc. 2005. V. 218. N 1. P. 68. doi 10.1111/j.13652818.2005.01457.x

[14] Wilson T. // J. Microsc. 2011. V. 244. N 2. P. 113. doi 10.1111/j.1365-2818.2011.03549.x

[15] Sheppard C.J.R., Gu M. // Appl. Opt. 1992. V. 31. N 14. P. 2541. doi 10.1364/AO.31.002541

[16] Hell S., Reiner G., Cremer C., Stelzer E. // J. Microsc. 1993. V. 169. N 3. P. 391. doi 10.1111/j.1365-2818.1993.tb03315.x

[17] Sheppard C.J.R., Gu M., Brain K., Zhou H. // Appl. Opt. 1994. V. 33. N 4. P. 616. doi 10.1364/AO.33.000616

[18] Wiersma S., Visser T., Török P. // Pure Appl. Opt. 1998. V. 7. P. 1237. doi 10.1088/0963-9659/7/5/029

[19] Izatt J.A., Hee M.R., Owen G.M., Swanson E.A., Fujimoto J.G. // Opt. Lett. 1994. V. 19. N 8. P. 590. doi 10.1364/OL.19.000590

[20] Dubois A., Moneron G., Boccara C. // Opt. Commun. 2006. V. 266. N 2. P. 738. doi 10.1016/j.optcom.2006.05.016

[21] Sheppard C.J.R., Roy M., Sharma M.D. // Appl. Opt. 2004. V. 43. N 7. P. 1493. doi 10.1364/AO.43.001493

[22] Optical Coherence Tomography. Technology and Applications / Ed. by Drexler W., Fujimoto J.G. Berlin: Springer, 2008. $1326 \mathrm{p}$.

[23] De Groot P., Colonna de Lega X., Kramer J., Turzhitsky M. // Appl. Opt. 2004. V. 43. N 25. P. 4821. doi 10.1364/AO.43.004821

[24] Abdulhalim I. // Ann. Phys. 2012. V. 524. N 12. P. 787. doi 10.1002/andp.201200106

[25] Gao W. // J. Mod. Opt. 2015. V. 62. N 21. P. 1764. doi 10.1080/09500340.2014.952689

[26] Labiau S., David G., Gigan S., Boccara A.C. // Opt. Lett. 2009. V. 34. N 10. P. 1576. doi 10.1364/OL.34.001576
[27] Grebenyuk A.A., Ryabukho V.P. // Opt. Lett. 2012. V. 37. N 13. P. 2529. doi 10.1364/OL.37.002529

[28] Лякин Д.В., Рябухо В.П. // Квант. электрон. 2013. Т. 43. B. 10. C. 949; Lyakin D.V., Ryabukho V.P. // Quantum Electron. 2013. V. 43. N 10. P. 949. doi 10.1070/QE2013v043n10ABEH015187

[29] Safrani A., Abdulhalim I. // Appl. Opt. 2011. V. 50. N 18. P. 3021. doi 10.1364/AO.50.003021

[30] Tearney G.J., Brezinski M.E., Southern J.F., Bouma B.E., Hee M.R., Fujimoto J.G. // Opt. Lett. 1995. V. 20. N 21. P. 2258. doi 10.1364/OL.20.002258

[31] Watanabe Y., Yamaguchi I. // Appl. Opt. 2002. V. 41. N 13. P. 2414. doi 10.1364/AO.41.002414

[32] Diaspro A., Federici F., Robello M. // Appl. Opt. 2002. V. 41. N 4. P. 685. doi 10.1364/AO.41.000685

[33] Visser T., Oud J., Brakenhoff G. // Optik. 1992. V. 90. N 1. P. 17.

[34] Лякин Д.В., Максимова Л.А., Сдобнов А.Ю., Рябухо В.П. // Опт. и спектр. 2017. Т. 123. № 3. С. 463; Lyakin D.V., Maksimova L.A., Sdobnov A.Yu., Ryabukho V.P. // Opt. Spectrosc. 2017. V. 123. N 3. P. 487. doi 10.1134/S0030400X17090235

[35] Wiersma S., Török P., Visser T., Varga P. // J. Opt. Soc. Am. A. 1997. V. 14. N 7. P. 1482. doi 10.1364/JOSAA.14.001482

[36] Рябухо В.П., Хомутов В.Л., Лякин Д.В., Константинов К.В. // Письма в ЖТФ. 1998. Т. 24. № 4. С. 19; Ryabukho V.P., Khomutov V.L., Lyakin D.V., Konstantinov K.V. // Technical Physics Letters. 1998. V. 24. N 2. P. 132. doi 10.1134/1.1262023

[37] Heil J., Heuck H.M., Müller W., Netsch M., Wesner J. // Appl. Opt. 2012. V. 51. I. 15. P. 3059. doi 10.1364/AO.51.003059

[38] Srivastava V., Nandy S., Mehta D.S. // Appl. Phys. Lett. 2013. V. 103 I. 10. P. 103702. doi 10.1063/1.4820350

[39] Zhou R., Jin D., Hosseini P., Singh V.R., Kim Y.-H., Kuang C., Dasari R.R., Yaqoob Z., So P.T.C. // Opt. Express. 2017. V. 25. N 1. P. 130. doi 10.1364/OE.25.000130

[40] Singla N., Srivastava V., Mehta D.S. // J. Biophotonics. 2018. V. 11. N 5. P. e201700279. doi 10.1002/jbio.201700279

[41] Электронный ресурс. Режим доступа: $\mathrm{https} / / /$ refractiveindex.info/?shelf=glass\&book=SCHOTTmultipurpose \&page $=$ D263TECO

[42] De Groot P., Colonna de Lega X. // Opt. Lett. 2007. V. 32. N 12. P. 1638. doi 10.1364/OL.32.001638.

[43] Ryabukho V.P., Lyakin D.V., Grebenyuk A.A., Klykov S.S. // J. Opt. 2013. V. 15. N 2. P. 025405. doi 10.1088/2040-8978/15/2/025405

[44] Рябухо В.П., Лычагов В.В., Лякин Д.В., Смирнов И.В. // Опт. и спектр. 2011. Т. 110. № 5. С. 854; Ryabukho V.P., Lychagov V.V., Lyakin D.V., Smirnov I.V. // Opt. Spectrosc. 2010. V. 110. N 5. P. 802. doi 10.1134/S0030400X11050134

[45] Korn G.A., Korn T.M. Mathematical handbook for scientists and engineers: definitions, theorems, and formulas for reference and review. New York: McGraw-Hill, 1968. 1150 p.; Корн Г., Корн Т. Справочник по математике (для научных работников и инженеров). М.: Наука, 1973. 832 с. 